\title{
SARS-COV-2 como desencadenante de enfermedades tromboembólicas en el embarazo
}

\section{SARS-COV-2 as triggering factor for thromboembolic diseases during pregnancy}

\author{
Kenneth Andersson Barrera García \\ Universidad San Carlos de Guatemala \\ kennethbg96@gmail.com \\ https://orcid.org/0000-0002-4202-3476
}

Recibido: $14 / 07 / 2021$ Aceptado: 01/09/2021

\section{Resumen}

\section{Referencia del artículo}

Barrera García, K. A. (2021). SARS-COV-2 como desencadenante de enfermedades tromboembólicas en el embarazo. Revista Diversidad Científica, 1(1). 159-167. DOI: https://doi.org/10.36314/diversidad.v1i1.17

OBJETIVO: Describir el SARS-COV-2 como desencadenante de enfermedades tromboembólicas en el embarazo MÉTODO: Se recopiló información de artículos de revistas científicas y metaanálisis. RESULTADOS: Durante la pandemia en el país de Chile se realizaron muestras de PCR, exámenes para detectar el virus $(89,2 \%)$ fueron mujeres gestantes. Se identificaron patologías asociadas como Hipertensión en el 9,7\% y pacientes con Diabetes Mellitus 1 o 2 en un $8.3 \%$, enfermedades tromboembólicas en un mismo porcentaje. CONCLUSIÓN: Actualmente, la infección por SARS-CoV-2 es una afección en la cual el daño endotelial y la interacción entre factores inflamatorios y tromboticos, posee una característica única, mediada por citoquinas y leucocitos, estableciendo una activación global en la cascada de coagulación, generando la trombosis masiva provocando tromboembolia pulmonar y trombosis venosa profunda, Se reconoce como un gran reto la prevención del Tromboembolismo Venoso, siempre se debe tener en cuenta, los 3 conceptos iniciales siguientes: "conocer factores de riesgo, realizar un diagnóstico oportuno e indicar el tratamiento profiláctico adecuado", todas estas gestiones están directamente relacionadas y como principal objetivo la disminución de la mortalidad materna.

Palabras clave: COVID-19, embarazo, paciente, trombofilia, tromboprofilaxis 


\begin{abstract}
TARGET: Describe the SARS-COV-2 as triggering factor for thromboembolic diseases during pregnancy. METHOD: Information was collected from articles in scientific journals and meta-analyzes. RESULTS: During the pandemic in the country of Chile, PCR samples were performed, tests to detect the virus (89.2\%) were pregnant women. Associated pathologies were identified as hypertension in $9.7 \%$ and patients with Diabetes Mellitus 1 or 2 in $8.3 \%$, thromboembolic diseases in the same percentage. CONCLUSION: Currently, SARS-CoV-2 infection is a condition in which endothelial damage and the interaction between inflammatory and thrombotic factors have a unique characteristic, mediated by cytokines and leukocytes, establishing a global activation in the coagulation cascade. , generating massive thrombosis causing pulmonary thromboembolism and deep vein thrombosis, The prevention of Venous Thromboembolism is recognized as a great challenge, the following 3 initial concepts must always be taken into account: "know risk factors, make a timely diagnosis and indicate adequate prophylactic treatment ", all these steps are directly related and the main objective is the reduction of maternal mortality
\end{abstract}

Keywords: COVID-19, pregnancy, patient, thrombophilia, thromboprophylaxis 


\section{Introducción}

La pandemia por COVID 19, decretada por la Organización Mundial de la Salud en el mes de marzo del año 2020, es una enfermedad que se propaga rápidamente causada por SARS-COV-2, que causa múltiples afecciones a nivel respiratorio. El virus fue identificado por primera vez en China, específicamente en Wuhan, Hubei, en el mes de noviembre de 2019. "Esta infección predispone a las personas a sufrir diversas enfermedades, como afecciones a nivel circulatorio debido a la inflamación excesiva (tormenta de citoquinas), activación de plaquetas, daño endotelial y cambios a nivel sanguíneo" (Grand, 2020, p. 51).

"El aumento de la mortalidad en estos pacientes se debe a un incremento de dímero-D." La cual se considera como un trastorno de coagulación asociada a COVID-19 (CAC). En el período de la mujer embarazada la infección por COVID-19 es un verdadero reto para los servicios de salud, ya que se suma a la lista de riesgos tromboembólicos que sufren las pacientes con normalidad. En Argentina se presentó el primer caso en marzo 2020, conforme avanzó el tiempo se presentó un incremento en la cantidad de pacientes COVID-19 positivos, llegando a pacientes embarazadas que son la población objetiva de los estudios que se citan en este ensayo (Grand, 2020, p. 53).

En la etapa del embarazo se presentan distintos signos y síntomas como, estasis sanguínea, daño endotelial e hipercoagulabilidad. Esto sucede debido al poco tiempo o pocos estudios acerca de trombo profilaxis en pacientes gestantes con COVID-19. Estudios anteriores han trabajado con base a lo reportado en publicaciones. "En el caso de mujeres con embarazo y diagnóstico de COVID 19, hospitalizadas, es recomendable iniciar terapia de tromboprofilaxis con $40 \mathrm{mg} / \mathrm{día}$ de Enoxaparina o Heparina no fraccionada en el tercer trimestre de embarazo" (Grand, 2020, p. 55).

La prolongación de la trombo profilaxis en pacientes ambulatorios que continúen su embarazo o en períodos postparto requieren de una estratificación con base a sus factores de riesgo y realizar una evaluación para observar la evolución del cuadro infeccioso. De esta manera, es sumamente importante considerar un cuidado durante el embarazo junto a todos los cambios en el sistema inmunológico. Este grupo específico de pacientes "posee mayor riesgo a infecciones virales y complicaciones más graves." En la actualidad, el contexto de SARS-CoV-2 se comprobó que las pacientes pueden desarrollar afección en vías respiratorias hasta un caso de neumonía grave que se asocian a malestares respiratorios progresivos (Grand, 2020, p. 58). 
La importancia que tiene el estudio del desarrollo de la enfermedad tromboembólica en mujeres embarazadas, radica en este estudio que tiene como objetivo principal determinar si el SARS-COV-2 es un desencadenante de enfermedad tromboembólica en el embarazo, que se realizó por medio de revisiones bibliográficas con el fin de conocer la fisiopatología de la Tromboembolia Pulmonar (TEP) y su relación en la etapa de gestación" (Cortés, 2020, p. 35).

\section{Contenido}

Uno de los grupos considerados de alto riesgo frente a los brotes de enfermedades infecciosas se encuentra en la población de mujeres embarazadas y sus fetos. Sus cambios fisiológicos durante esta etapa muestran un incremento a la "susceptibilidad de infecciones, especialmente en el sistema cardiorrespiratorio." Como consecuencia, se observa que este grupo tiene mayor predisposición a enfermedades, el cual se puede relacionar con el incremento de la morbimortalidad materna (Grand, 2020, p. 59).

Las infecciones por coronavirus varían de forma independiente a los casos (leves a moderadas), pero las pacientes embarazadas siendo vulnerables durante esta etapa pueden contagiarse con el síndrome respiratorio agudo severo (causado por el SARS-CoV); en el que durante el embarazo ocasione una disminución de la inmunidad celular. "Los cambios fisiológicos e inmunológicos que se desarrollan en el embarazo aumentan el riesgo de estas pacientes y provocar graves infecciones por coronavirus" (Cobo, 2021, p. 7).

En el período de embarazo, existen cambios a nivel respiratorio y circulatorio, produciendo en ocasiones rinorrea y disnea fisiológica que es causada por los cambios en los volúmenes respiratorios y el aumento de la demanda de oxígeno para el feto. Es importante diferenciarla de la disnea patológica ya que ésta puede indicarnos una neumonía derivada de la enfermedad COVID-19. "Un retraso en el diagnóstico de esta enfermedad agravaría la situación e incrementaría el riesgo de que diese lugar a una insuficiencia respiratoria, entre otras complicaciones" (Cobo, 2021, p. 9).

Una de las características clínicas principales de la enfermedad por COVID-19 es que se observa una infección a nivel respiratorio. Un alto porcentaje de estas pacientes avanza hacia una enfermedad más severa debido a una infección sistémica. El daño pulmonar es ocasionado por la respuesta exagerada del virus a nivel respiratorio agudo, produciendo shock y disfunción multiorganica. El riesgo en esta 
población son los trastornos a nivel circulatorio. En un elevado porcentaje el Tromboembolismo Venoso (TEV) no registra antecedentes de eventos previos de TEV ni trombofilias" (Grand, 2020, p. 60).

Al mencionar otros elementos de riesgo, se puede establecer que uno de los más cruciales por su elevada prevalencia, es la obesidad. La Organización Mundial de la Salud define la obesidad como una acumulación anormal o excesiva de grasa que puede perjudicar a la salud y se detalla operativamente cuando el Índice de Masa Corporal (IMC) es $\geq 30 \mathrm{~kg} / \mathrm{m} 2$. "Al tomar este factor en cuenta, el riesgo de TEV crece conforme el aumento del Índice de Masa Corporal por encima de 25 kg/m2" (Grand, 2020, p. 61).

"Los pacientes contagiados por el virus SARS-CoV-2 se encuentran con un riesgo incrementado de Tromboembolia Venosa." Es desconocido si este riesgo es significativo o superior al de nuevas infecciones, a pesar de los estudios en el periodo de embarazo aún no se conoce con exactitud. Los elementos involucrados en las trombosis son diversos, pero entre ellos destaca: la inmunotrombosis, que es responsable de la trombosis a nivel de la microvasculatura pulmonar (Grand, 2020, p. 63).

"Las comorbilidades preexistentes como la edad materna alta, el Índice de Masa Corporal elevado, la Hipertensión Arterial y la Diabetes Mellitus parecen ser factores de riesgo para COVID-19." Otro dato que provoca inquietud es que la tasa de nacimientos prematuros en las mujeres embarazadas con COVID-19 son superiores que en las mujeres embarazadas sin la enfermedad (Tortosa, 2020, p. 2).

“Las manifestaciones clínicas pueden ser trombóticas o hemorrágicas." El embarazo añade mayor complejidad dada su hipercoagulabilidad fisiológica. Un aumento de factores de coagulación, incluyendo fibrinógeno y dímero $D$ aumentan hasta un $50 \%$ por encima del valor inicial en el tercer trimestre, convirtiendo a las pacientes en este periodo aún más vulnerables. Informes recientes colocan al dímero D elevado como indicador de pronóstico en personas no embarazadas que padecen COVID-19 (Tortosa, 2020, p. 3).

La fisiopatología que afecta la Tromboembolia Pulmonar con la infección por SARS-CoV-2 parece estar relacionada con trastornos en la coagulación. "La respuesta inflamatoria sistémica y la disfunción endotelial son asociados a la infección por el virus que lleva a un aumento en la formación de trombina combinado con una baja de los anticoagulantes naturales del organismo" (Calva , Carrasco, \& García, 2020, p. 924). 
La repercusión para tromboembolia pulmonar (TEP) en las pacientes embarazadas es mayor en comparación con la mujer no embarazada, su prevalencia es de 4,7 casos por cada 10.000 , con un porcentaje de 6,6\% de mortalidad. "La estasis venosa afecta en el primer trimestre del embarazo y se mantiene hasta llegar al tercer trimestre (Calva, Carrasco, \& García, 2020, p. 926).

Según investigadores indican que en sus estudios realizados en 2020 por primera vez, observaron cambios a nivel histológico y patológico en la placenta de mujeres con embarazo tardío y diagnóstico de COVID-19. "El tejido analizado a nivel de la placenta presentó depósitos de fibrina e infartos, lo que muestra un resultado de una hipoperfusión placentaria debido a la presencia de receptores ECA II en la placenta (Domínguez, 2020, p. 399).

En el estudio observado, en el que se tomaron diversos documentos de carácter descriptivo, en Hospital de la Ciudad de Chile en donde se reportaron casos de pacientes obstétricas diagnosticadas con COVID 19 con un alto porcentaje de $89,2 \%$. Dentro de este grupo de pacientes, un poco más de la mitad corresponden a mujeres asintomáticas, lo cual condiciona a los estudios realizados. La mayoría de las pacientes requirió un manejo básico de la patología y sólo 7 pacientes requirieron cuidados intensivos. Asociadas a estas pacientes también se encontraron patologías de base como hipertensión y diabetes mellitus 1 y 2 en distintos porcentajes 9,7 y $8,3 \%$ (Morales, 2020, p. 48).

Dentro de las complicaciones observadas en el estudio antes mencionado se exhibió trastornos de vena cava inferior e iliaca derecha en una paciente. Se debe recalcar el proceso de implementación temprana de las "medidas de trombo profilaxis a todas las pacientes diagnosticadas positivas en el tercer periodo de embarazo y puerperio, se prefiere que sea con un régimen de 10 días de heparina fraccionada a aquellas con casos asintomáticos o leves; y 6 semanas a aquellas con casos graves (Morales, 2020, p. 50).

\section{Conclusiones}

Actualmente, se ha constatado que la infección por SARS-CoV-2, es un desencadenante de enfermedades tromboembólicas en el embarazo debido a los cambios fisiológicos y mecánicos que se observan durante la gestación, en donde el daño endotelial, la interacción entre factores inflamatorios y tromboticos, poseen una característica única, mediada por citoquinas y leucocitos, estableciendo una activación global en la cascada de coagulación, generando la trombosis masiva 
provocando tromboembolia pulmonar y trombosis venosa profunda (Samaniego \& Conte, 2020, p. 1467).

Se reconoce como un gran reto la prevención del Tromboembolismo Venoso, siempre se debe tener en cuenta, los 3 conceptos iniciales siguientes: "conocer factores de riesgo, realizar un diagnóstico oportuno e indicar el tratamiento profiláctico adecuado. Todas estas gestiones están directamente relacionadas y como principal objetivo la disminución de la mortalidad materna (Domínguez, 2020, p. 400).

El monitoreo permanente de los distintos factores de riesgo (preexistentes o de aparición durante el curso del embarazo) puede ayudar a identificar a los grupos de riesgo e indicar de manera temprana la profilaxis farmacológica con heparinas fraccionadas. Debido que el COVID-19 tiene una rápida propagación y ha puesto en alerta a toda la comunidad médica y de la salud" sobre el incremento del riesgo trombótico a la mujer embarazada y población mundial (Grand, 2020, p. 70).

\section{Declaración}

El estudio se realizó con fines médicos, de acuerdo al Código de Ética y Buenas Prácticas COPE.

\section{Conflicto de intereses}

El autor declara no tener ningún conflicto de intereses.

\section{Referencias}

Calva , M., Carrasco, C., \& García, N. (2020). Tromboembolia pulmonar como complicación de COVID-19 en el puerperio. Reporte de un caso. Revista Chilena de Anestesia, 924-929. https://doi.org/10.25237/revchilanestv49n06-21

Cobo, M. (2021). Embarazo y Sistema Inmunitario en Tiempos de Pandemia. Obtenido de Universidad de Cantabria: https://repositorio.unican.es/xmlui/bitstream/ handle/10902/22158/COBO\%20BLANCO,\%20MARIA.pdf?sequence=1 
Cortés, J. (2020). Embarazo, recién nacido y COVID-19. Medicina Balear, 35-38. http://www.medicinabalear.org/pdfs/Vol35n3.pdf

Díaz, J. (2020). Clinical-epidemiological features in 36 Cuban children with COVID-19. Revista Cubana de Pediatría, 399-406. http://scielo.sld.cu/scielo. php?script=sci_arttext\&pid=S0034-75312020000500004

Domínguez, R. (2020). Enfermedad por coronavirus 2019. Acta Médica, Grupo Ángeles, 399-406. https://doi.org/10.35366/97267

Grand, B. (2020). Profilaxis del Tromboembolismo Venoso en Embarazo y Puerperio: Actualización en tiempos de infección por COVID-19. Revista Hematología, 5170. https://revistahematologia.com.ar/index.php/Revista/article/view/306

Morales, N. (2020). Pandemia SARS-CoV-2 y embarazo en el Hospital el Pino: un estudio descriptivo. https://doi.org/10.4067/S0717-75262020000700008

Samaniego, F., \& Conte, G. (2020). Infección por SARS-CoV-2. Una nueva enfermedad endotelial trombo-inflamatoria. https://doi.org/10.4067/ S0034-98872020001001467

Tortosa, F. (2020). Riesgo materno fetal y uso de tromboprofilaxis para prevención de enfermedad tromboembólica en la mujer embarazada. Rio Negro. https://www. researchgate.net/publication/347483918_Riesgo_materno_fetal_y_uso_de_ tromboprofilaxis_para_prevencion_de_enfermedad_tromboembolica_en_la_ mujer_embarazada_Resumen_de_evidencia_y_evaluacion_de_eficacia_y_ seguridad

\section{Sobre el autor \\ Kenneth Andersson Barrera García}

Es es tudiante de la carrera de médico y cirujano, participante en la investigación "Caracterización Clínico-Epidemiológica de Traumatismo Craneoencefálico Pediátrico" y de la investigación "Caracterización Clínica Y Epidemiológica de Hemorragia Obstétrica”. Carrera de médico y cirujano de la Universidad de San Carlos de Guatemala. 
Copyright (c) Kenneth Andersson Barrera García

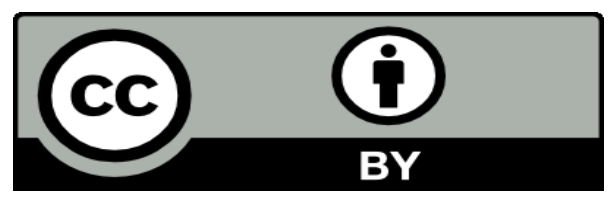

Este texto está protegido por una licencia CreativeCommons 4.0.

Usted es libre para compartir, copiar y redistribuir el material en cualquier medio o formato y adaptar el documento, remezclar, transformar y crear a partir del material para cualquier propósito, incluso comercialmente, siempre que cumpla la condición de atribución: usted debe reconocer el crédito de una obra de manera adecuada, proporcionar un enlace a la licencia, e indicar si se han realizado cambios. Puede hacerlo en cualquier forma razonable, pero no de forma tal que sugiera que tiene el apoyo del licenciante o lo recibe por el uso que hace. 